\title{
An electrochemical model of semi-conductive polymers
}

\author{
Hiroyuki Aizawa* \\ Aizawa Science Museum, Kasukabe, Saitama, Japan
}

Since discovery of semi-conductive organic polymers in 1970's, several hypotheses have been proposed for explanation of conductivity of the substances [1-4]. However, fundamental electric nature of the polymers remains unclear. For example, it is mystery that undoped polyacetylene shows dielectric nature under microwave exposure with high dielectric constant at around 1000, which is more than ten times higher than that of water [5]. Based on electronegativity theory, it is difficult to explain how carbohydrate compound could make polar residues for dielectric property [6]. Moreover, graphene is conductive without doping, although the doping theory suggests that $\pi$-electron should flow only when the dopants accept or donate $\pi$-electrons from double bonds [7]. We need to explain the inconsistency in order to understand fundamental mechanisms of conductivity of polymers. Here, we would propose a novel theory of conductivity from electrochemical point of view. In electrochemical reactions such as electrolysis, electric current corresponds to a chain reaction of transfer of chemical bonds, which are formed by an electron pair or magnetic attraction between atoms. In other words, electric current is a wave of magnetic re-pairing along the chain of chemical bonds.

Valence theory of chemical nature of atoms is based on a consumption that rotation of an electron in an orbital around the atomic nucleus causes magnetic field around the atom. If two neighboring atoms have anti-parallel magnetic fields, the atoms bind to each other covalently by association of the magnets side-by-side. In other words, electrons with up- and down-spin, respectively, attract each other between two adjacent atoms forming an electron pair. Accordingly, the electron pair corresponds to a covalent bond, which binds two atoms in a molecule. This basic and simple idea came from theoretical atomic structure in which electrons rotate around an atomic nucleus early in the $20^{\text {th }}$ century [6]. On the other hand, if we hypothesize that atoms consist of magnets, which should correspond to a rotation of an electron, we do not have to take account of a repulsive force between two electrons in a chemical bond.

In chemistry, we usually use a chemical formula in which an antiparallel pair of magnets are referred to a dash between elements such as $\mathrm{H}-\mathrm{O}-\mathrm{H}$ for a water molecule [6]. In this formula, we may also describe the water molecule using arrows of magnets as follows: $\mathrm{H} \uparrow \downarrow \mathrm{O} \uparrow \downarrow \mathrm{H}$. During chemical reactions, some chemical bonds are broken and reformed to form products. During this chemical reaction, each atom retains its magnets, and it changes its pairing partner, for example, as follows:

$$
\mathrm{H} \uparrow \downarrow \mathrm{H}+\mathrm{Cl} \uparrow \downarrow \mathrm{Cl} \leftrightarrow \mathrm{H} \uparrow \downarrow \mathrm{Cl}+\mathrm{Cl} \uparrow \downarrow \mathrm{H}
$$

Poly-acetylene is one of semi-conductive polymers, whose chemical formula omitting hydrogen is as follows:

$$
-\mathrm{C}=\mathrm{C}-\mathrm{C}=\mathrm{C}-\mathrm{C}=\mathrm{C}-\text { or }-\mathrm{C} \uparrow-\downarrow \mathrm{C}-\mathrm{C} \uparrow-\downarrow \mathrm{C}-\mathrm{C} \uparrow-\downarrow \mathrm{C}-
$$

In this formula, a single- and double-bars represent a single and double bond, respectively, and an arrow represents a magnet that forms anti-parallel pair with that of the neighboring atom. It is possible to represent all the chemical bonds as pairs of magnets, if required. When a current flow along the polyacetylene, the chemical bonds become shifted as follows:

\section{$=\mathrm{C}-\mathrm{C}=\mathrm{C}-\mathrm{C}=\mathrm{C}-\mathrm{C}=$ or $-\uparrow \mathrm{C}-\mathrm{C} \downarrow-\uparrow \mathrm{C}-\mathrm{C} \downarrow-\uparrow \mathrm{C}-\mathrm{C} \downarrow-$}

During the electric current flow, each magnetic pair dissociates and re-associates with a magnet of the other neighbor carbon atom to form a new magnetic pair. In other words, an electric current corresponds to a wave of magnetic coupling. The wave of magnetic coupling propagates as a flow of electron transfer reactions as shown in Figure 1. The current flows in the opposite direction to that of electron transfer reactions.

Semi-conductive organic polymers have some limited molecular mass at around 40,000. While some of them take an open circular form, others form closed ring-shaped macromolecules. Since they are semi-conductive within molecules, closed circular polymers could flow alternating currents as microcircuit current when exposed to microwave. The alternating circular current produces alternating magnetic field to cancel that of microwave. Although the alternating circular current flows within a microcircuit of the polymer, the current cannot leak outside. Thus, polyacetylene is an insulator before doping. On the other hand, doping could induce leaks of electric currents flow

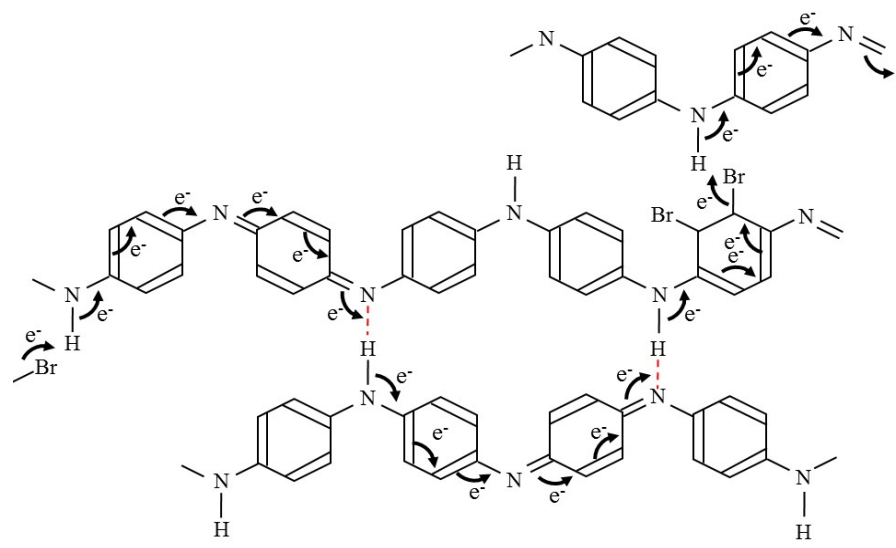

Figure 1. Electrochemical model for electric current in polyaniline molecules doped with bromide. An arrow indicates transfer of chemical bond. e; an electron. A dashed red line indicates a hydrogen bond

${ }^{*}$ Correspondence to: Hiroyuki Aizawa, Aizawa Science Museum, 1-10-13 Yutaka-cho, Kasukabe, Saitama, 3440066, Japan, Tel: +81-48-754-9880, E-mail: aizawa@rr.iij4u.or.jp

Received: June 04, 2019; Accepted: June 25, 2019; Published: June 26, 2019 
among semi-conductive polymers (Figure 1). Accordingly, conductivity of the whole polymer material should be dramatically increased by the leaks. In case of polyaniline, not only doping but also hydrogen bonds between molecules work effectively for transfer of electric currents between molecules (Figure 1). This is the reason why electrochemical polymerization works well to form a film of polyaniline, which has semi-conductivity even without doping.

Open circular form of semi-conductive polymers could not flow electric current without doping or hydrogen bonds. Accordingly, microwave exposure induces alternating electric motivation in the open circular polymers, resulting in oscillation of positive and negative charges at their both ends. The frequency characteristics of the dielectric materials depend on the length of the polymer. Since dopants leak electric current among molecules, the dielectric nature of undoped polymers under microwave should be lost by doping.

Figure 1 shows an electrochemical molecular model of electric current in polyaniline doped with bromide, which could react with $\pi$-electrons of benzene ring. Polyaniline is a well-known conductive polymer. In this model, electric current flows as a chain reaction of transfer of chemical bonds. Within a single polyaniline molecule, $\pi$-electrons in conjugated double bonds play a central role for current flow, while $\sigma$-electron of hydrogen, which forms covalent and hydrogen bonds among nitrogen atoms of two adjacent polyaniline molecules, plays a pivotal role for a current leak between the polyaniline molecules. Since the hydrogen transfer reaction between molecules is reversible, alternating current could flow along this current path. On the contrary, doped bromides are released from the molecules by current flow binding to hydrogen of neighboring polyaniline molecule, which induces current flow in the neighboring polyaniline molecule (Figure 1). In this reaction, electrons flow from donor to acceptor in a unidirectional manner, which may be a fundamental principle of semiconductive rectifier diodes. Since the resultant hydrobromide is a gas, it diffuses randomly to react with benzene ring of polyaniline molecules nearby, modifying chemical structure of polyaniline by re-doping.
Since this re-doping reaction is not necessarily reversible, this reaction is suitable for direct current of diodes but not for alternating current.

Semi-conductive polymer is useful for electronical equipment and implant materials in medical and surgical treatments for patients. For example, some semi-conductive gel has been used as electrode materials for electroencephalography and electro-cardiograph. It is also possible that artificial nerve fibers are assembled from semi-conductive polymers, which could cure patients with neurodegenerative diseases or neuronal damages by spine injury. I hope that the theory proposed here stimulates research and development of conductive polymers especially for medical application in near future.

\section{Acknowledgement}

I would like to express my special thanks to organizing committee of $5^{\text {th }}$ Global Congress and Expo on Materials Science and Engineering held at Osaka on Jun 10-11, 2019, for encouragement and discussion on electrochemistry and semi-conductive polymers.

\section{References}

1. Shirakawa H, Louis EJ, MacDiarmid AG, Chiang CK, Heeger AJ (1977) Synthesis of electrically conducting organic polymers: Halogen derivatives of polyacetylene, $(\mathrm{CH})$ x. J Chem Soc Chem Comm 578-580.

2. Ito T, Shirakawa H, Ikeda S (1974) Simultaneous polymerization and formation of polyacetylene film on the surface of concentrated soluble Ziegler-type catalyst solution. J Polym Sci Polym Chem 12: 11-20.

3. Chiang CK, Fischer CR, Park YW, Heeger AJ, Shirakawa H, et al. (1977) Electrical conductivity in doped polyacetylene. Phys Rev Letters 39: 1098.

4. Chiang CK, Druy MA, Gau SC, Heeger AJ, Louis EJ, et al. (1978) Synthesis of highly conducting films of derivatives of polyacetylene, (CH)x. J Am Chem Soc 100: 1013.

5. Gamoudi M, Andre JJ, Francois B, Maitrot M (1982) Dielectric properties of undoped poly-acetylene fims in a large frequency range. $J$ Phys 43: 953-959.

6. Pauling L (1970) General Chemistry, Dover Publications; pp151.

7. Winokur M, Moon YB, Heeger AJ, Barker J, Bott DC, et al. (1987) X-Ray scattering from sodium-doped polyacetylene: Incommensurate-commensurate and order-disorder transformations. Phys Rev Letters 58: 2329.

Copyright: (C2019 Aizawa H. This is an open-access article distributed under the terms of the Creative Commons Attribution License, which permits unrestricted use, distribution, and reproduction in any medium, provided the original author and source are credited. 